\title{
Uniqueness of Flat Spherically Symmetric Spacelike Hypersurfaces Admitted by Spherically Symmetric Static Spactimes
}

\author{
Robert Beig $^{1}$ and Azad A. Siddiqui ${ }^{2}$ \\ ${ }^{1}$ Gravitational Physics, Faculty of Physics, University of Vienna, A-1090 Vienna, \\ Austria, E-mail: Robert.Beig@univie.ac.at \\ ${ }^{2}$ Department of Basic Science and Humanities, EME College, National University of \\ Science and Technology, Peshawar Road, Rawalpindi, Pakistan, \\ E-mail: azad@ceme.edu.pk
}

\begin{abstract}
It is known that spherically symmetric static spacetimes admit a foliation by flat hypersurfaces. Such foliations have explicitly been constructed for some spacetimes, using different approaches, but none of them have proved or even discussed the uniqueness of these foliations. The issue of uniqueness becomes more important due to suitability of flat foliations for studying black hole physics. Here flat spherically symmetric spacelike hypersurfaces are obtained by a direct method. It is found that spherically symmetric static spacetimes admit flat spherically symmetric hypersurfaces, and that these hypersurfaces are unique up to translation under the timelike Killing vector. This result guarantees the uniqueness of flat spherically symmetric foliations for such spacetimes.
\end{abstract}


PACS: 04.20.-q, 04.20.Ex, 04.20.Gz

\section{Introduction}

Splitting a space into a sequence of subspaces, such that every point in the space lies in one and only one of the subspaces, is called a foliation. The foliation of an $n$ dimensional manifold, $M$, is a decomposition of $M$ into submanifolds, all being of the same dimension, $p$. The submanifolds are the leaves of the foliation. The co-dimension, $q$, of a foliation is defined as $q=n-p$. A foliation of co-dimension one is called a foliation by hypersurfaces. The simplest and best understood cases of foliation are when $p=q=1$, e.g. the two dimensional xy-plane, $\mathbb{R}^{\not}$, which can be foliated by the straight lines, $y=m x+c$, with $c$ taken as the parameter and any fixed $m$. Notice that a foliation of the of xy-plane by straight lines is not unique, as different 'fixed' values of $m$ will give different sequences of foliating straight lines with a different slope.

In General Relativity (GR) one often requires to use a sequence of spacelike or null hypersurfaces to foliate the spacetime. There has been a lot of work to obtain foliations by hypersurfaces of zero mean extrinsic curvature called 'maximal slicing' [1]-[4] and by hypersurfaces of constant mean extrinsic curvature known as 'CMC-slicing' [4]-[11]. There has also been significant work on foliations by hypersurfaces of zero intrinsic curvature called 'flat foliations' [12]-[18]. Existence of flat spacelike foliations for spherically symmetric static spactimes (SSSS) is shown via the Hamiltonian equations of general relativity in [14] and using an initial value approach in [15], [16]. Complete foliations of the Schwarzschild and Reissner Nordström (RN) spacetimes by flat space- 
like hypersurfaces is also obtained using the fact that the normals to such foliations are geodesics [17], [18]. Being indirect approaches, earlier procedures do not guarantee the uniqueness of these foliations. As a flat foliation covers the most interesting regions of spacetime describing realistic gravitational collapse it is specially suited for studying Hawking radiation from a fully quantum gravitational viewpoint. Husain and Winkler [19] have presented a flat slice Hamiltonian formalism to have "a standard model" for studying black hole physics. The non-uniqueness of flat foliations may raise the question on the validity of the results if a different sequence of flat slices is used in their model.

In this paper, in order to obtain flat spacelike hypersurfaces, we use the direct approach (i.e. solve $R_{j k l}^{i}=0$, where $R_{j k l}^{i}$ are the components of the Riemann curvature tensor for the hypersurfaces). Solution of the above system gives a unique sequence of flat spherically symmetric spacelike hypersurfaces admitted by SSSS, thus showing the uniqueness of flat spherically symmetric foliations for such spacetimes. In the following sections, after presenting solution of the equations giving flat spherically symmetric hypersurfaces and some examples, a conclusion is given. 


\section{Flat Spherically Symmetric Hypersurfaces Ad- mitted by Spherically Symmetric Static Space- times}

The most general form of a spherically symmetric static spacetime metric in the usual coordinates is

$$
d s^{2}=e^{v(r)} d t^{2}-e^{\lambda(r)} d r^{2}-r^{2} d \Omega^{2}
$$

where

$$
d \Omega^{2}=d \theta^{2}+\sin ^{2} \theta d \phi^{2}
$$

Now take an arbitrary hypersurface, $f(t, r, \theta, \phi)=0$. Considering spherical symmetry, taking $\theta$ and $\phi$ constant, this hypersurface in explicit form can be given as

$$
t=F(r)
$$

The induced 3-metric (of the hypersurfaces) is then

$$
d s_{3}^{2}=-\left(e^{\lambda(r)}-e^{v(r)} F^{\prime 2}\right) d r^{2}-r^{2} d \Omega^{2}
$$

For the induced metric to be flat a necessary but not sufficient condition, namely the Ricci scalar $=R=0$, implies

$$
\frac{r\left(-\lambda^{\prime} e^{\lambda}+\nu^{\prime} e^{\nu} F^{\prime 2}+2 e^{\nu} F^{\prime} F^{\prime \prime}\right)}{\left(e^{\lambda}-e^{\nu} F^{\prime 2}\right)^{2}}+\frac{1-e^{\lambda}+e^{\nu} F^{\prime 2}}{e^{\lambda}-e^{\nu} F^{\prime 2}}=0
$$

where' represents the derivative with respect to $r$. Using the substitution

$$
g^{2}(r)=\frac{1}{e^{\lambda}-e^{\nu} F^{\prime 2}}
$$


Eq.(5) becomes

$$
2 r g g^{\prime}+g^{2}-1=0
$$

and we have the general solution

$$
g^{2}(r)=1-\frac{c}{r}
$$

where $c$ is an arbitrary constant with dimensions of length. The induced metric now takes the form

$$
d s_{3}^{2}=-\frac{d r^{2}}{1-\frac{c}{r}}-r^{2} d \Omega^{2} .
$$

The above metric, Eq.(91), of the hypersurfaces is flat, i.e. all the components of the Riemann curvature tensor are zero (which is the necessary and sufficient condition for the hypersurfaces to be flat), only if $c=0$ or in other words only if

$$
g^{2}(r)=1 .
$$

Then, from Eqs.(3) and (6) , the flat spherically symmetric hypersurfaces are uniquely given as

$$
t=F(r)=\int e^{\frac{\lambda-\nu}{2}} \sqrt{1-e^{-\lambda}} d r .
$$

The mean extrinsic curvature, $K$, of these hypersurfaces is

$$
K=e^{\left(\frac{\nu+\lambda}{2}\right)}\left(\frac{\nu^{\prime} e^{\nu}}{2 \sqrt{1-e^{\nu}}}-\frac{2 \sqrt{1-e^{\nu}}}{r}\right)
$$

and the Hamiltonian constraint gives

$$
R+K^{2}-K_{a b} K^{a b}=\frac{2\left(K^{2}-e^{\nu}\right)}{r^{2}}-\frac{2 \nu^{\prime} e^{\nu}}{r},
$$

(here for flat hypersurfaces $R=0$ ). 


\section{Some Examples}

For the exterior Schwarzschild spacetime, given by the metric in Eq.(1) with $e^{v(r)}=$ $e^{-\lambda(r)}=1-2 m / r$ where $m$ is the mass, solution of Eq.(11) provides the unique sequences of flat spherically symmetric spacelike hypersurfaces

$$
t=F(r)=t_{c}-4 m \sqrt{\frac{r}{2 m}}-2 m \ln \left|\frac{\sqrt{\frac{r}{2 m}}-1}{\sqrt{\frac{r}{2 m}}+1}\right|,
$$

where $t_{c}$ is an integration constant which gives the time of the hypersurface i.e. the distinct values of $t_{c}$ correspond to the distinct flat hypersurfaces. Notice that the expression in Eq.(13) is same as the Lemaittre coordinates (see, e.g., [20]) for the Schwarzschild geometry or the flat hypersurfaces obtained by using the fact that these hypersurfaces are orthogonal to the unforced geodesics in [18]. The mean extrinsic

curvature, $K$, of these hypersurfaces is $3 \sqrt{\frac{m}{2 r^{3}}}$. The Hamiltonian constraint in this case gives $R+K^{2}-K_{a b} K^{a b}=0$.

The exterior Reissner-Nordstrom spacetime is given by the metric in Eq.(1) with $e^{v(r)}=$ $e^{-\lambda(r)}=1-2 m / r+Q^{2} / r^{2}$ where $m$ and $Q$ represent mass and charge respectively. In the case when $Q>m$ solution of Eq.(11) gives

$t=F(r)=t_{c}-2 E(r)-m \ln \left|\frac{r-E(r)}{r+E(r)}\right|-\frac{2 m^{2}-Q^{2}}{\sqrt{Q^{2}-m^{2}}}\left[\tan ^{-1}\left(\frac{E(r)-m}{\sqrt{Q^{2}-m^{2}}}\right)+\tan ^{-1}\left(\frac{E(r)+m}{\sqrt{Q^{2}-m^{2}}}\right)\right]$

where

$$
E(r)=\sqrt{2 m r-Q^{2}}
$$


and $t_{c}$ is the constant of integration. For $Q<m$ we have

$t=F(r)=t_{c}-2 E(r)-m \ln \left|\frac{r-E(r)}{r+E(r)}\right|-\frac{2 m^{2}-Q^{2}}{\sqrt{m^{2}-Q^{2}}} \ln \left[\frac{m r-E(r) \sqrt{m^{2}-Q^{2}}-Q^{2}}{m r+E(r) \sqrt{m^{2}-Q^{2}}-Q^{2}}\right]$,

and for the extreme case i.e. $Q=m$ we have

$$
t=F(r)=t_{c}-2 E(r)+\frac{m E(r)}{r-m}+4 m \tanh ^{-1}\left[\frac{E(r)}{m}\right]
$$

The mean extrinsic curvature, $K$, of the flat hypersurfaces in all cases of the RN

spacetime is $\frac{3 m r-Q^{2}}{r^{2} \sqrt{2 m r-Q^{2}}}$ and the Hamiltonian constraint gives $R+K^{2}-K_{a b} K^{a b}=\frac{2 Q^{2}}{r^{4}}$.

\section{Conclusion}

There has been lot of work on existence and construction of foliation of SSSS by spacelike hypersurfaces of zero intrinsic curvature. Perhaps, assuming the difficulty to solve the system of differential equations, $R_{j k l}^{i}=0$, in all earlier works indirect approaches have been used. In this paper, in order to obtain all possible sequences of flat spherically symmetric hypersurfaces admitted by SSSS, we have solved this system of differential equations. It is found that there exists a unique sequence of flat spherically symmetric spacelike hypersurfaces admitted by SSSS, guaranteeing the uniqueness of foliation by these hypersurfaces for such spacetimes. To emphasize the point, it is not just that the foliation of SSSS by flat spherically symmetric spacelike hypersurfaces is unique, but that these spacetimes admit a unique sequence of flat spherically symmetric spacelike hypersurfaces which form a foliation. Notice that the flat spherically symmetric spacelike hypersurfaces can also be obtained simply by changing the sign in Eq.(3) and in 
expressions for extrinsic curvatures. This corresponds to the hypersurfaces orthogonal to the incoming instead of outgoing geodesics [18].

In this paper we have studied flat slices of static, spherically symmetric spacetimes, where the slices themselves are also assumed to have spherical symmetry. Dropping the latter restriction would result in a study of a system of partial differential equations for the height function, as opposed to the ordinary differential equation, Eq.(15), of the slice. This is outside the scope we have set ourselves here.

\section{Acknowledgments}

One of the authors AAS highly appreciates role of the Higher Education Commission of Pakistan and is thankful for the financial support during the post doctoral research in Sweden during which this work was initiated. Authors are also grateful to the two referees for their comments which have improved quality of the paper.

\section{References}

[1] F. Estabrook, H. Wahlquist, S. Christensen, B. DeWitt, L. Smarr and E. Tsiang, Phys. Rev. D 7(1973)2814.

[2] J.E. Marsden and F.J. Tipler, Phys. Reports 66(1980)109.

[3] R. Beig and N.O'. Murchadha, Phys. Rev. D 57(1998)4728. 
[4] L. Smarr and J.W. York Jr., Phys. Rev. D 17(1978)2529; D.M. Eardley and L. Smarr, Phys. Rev. D 19(1978)2239; A. Lichnerowich, J. Math. Pure Appl. 23(1944)37; J. York, Phys. Rev. Letters 28(1972)1082; A.A. Siddiqui, Some Foliations of Black Hole Spacetimes, Ph.D Thesis, Quaid-i-Azam University, Islamabad, Pakistan(2000).

[5] A. Goddard, Spacelike surfaces of Constant Mean Curvature, Ph.D. thesis, Oxford University (1975); Commun. Math. Phys. 54(1977)279; Math. Proc. Cambridge Philos. Soc. 82(1977)489; Gen. Rel. Grav. 8(1977)525.

[6] D.R. Brill and F. Flaherty, Commun. Math. Phys. 50(1976)157; Ann. Inst. Henri Poincare 28(1978)335; D.R. Brill, J.M. Cavallo and J.A. Isenberg, J. Math. Phys. $21(1980) 2789$.

[7] A. Qadir and J.A. Wheeler, From SU(3) to Gravity; Yuval Neeman Festchrift, eds. E.S. Gotsman and G. Tauber (Cambridge University Press); Spacetime Symmetries, Proceedings of the Workshop, College Park, Maryland, 1988, edited by Y.S. Kim and W.W. Zachary [Nucl. Phys. B (Proc. Suppl.), 6(1989)345].

[8] A. Pervez, A. Qadir and A.A. Siddiqui, Phys. Rev. D 51(1995)4598.

[9] A.D. Rendall, Helv. Phys. Acta 69(1996)490.

[10] A. Qadir and A. A. Siddiqui, J. Math. Phys. 40(1999)5883.

[11] R. Beig and J.M. Heinzle, Commun. Math. Phys. 260(2005)673 (ArXiv: gr-qc/0501020, v2, 9 Feb. 2005).

[12] P. Kraus and F. Wilczek, Nucl. Phys. B433(1995)403. 
[13] S. Corley and T. Jacobson, Phys. Rev. D 57(1998)6269.

[14] V. Hussain, A. Qadir and A. A. Siddiqui, Phys. Rev. D 65(2002)027501 (ArXiv: gr-qc/0110068, v3, 20 Apr. 2002).

[15] M. Iriondo, E. Malec and N.O'. Murchadha, Phys. Rev. D 54(1996)4792 (ArXiv: gr-qc/9503030, v1, 16 Mar. 1995).

[16] J. Guven and N.O'. Murchadha, Phys. Rev. D 60(1999)104015.

[17] A. Qadir and A.A. Siddiqui, Nuovo Cimento B 117(2002)909.

[18] A. Qadir and A.A. Siddiqui, Int. J. Mod. Phys. D 15(2006)1419.

[19] V. Husain O. Winkler, Phys. Rev. D 71(2005)104001 (ArXiv: gr-qc/0503031, v2, 26 Apr. 2005).

[20] Yu. A. Rylov, Zh. Eksp. Teor. Fiz. 40(1961)868 [Sov. Phys. JETP 13(6)(1961)1235. 